\section{Differences in the dissipation of the effect of adaptation to two kinds of field displacement during head movements*}

\author{
HANS WALLACH and KARL JOSEF FREY \\ Swarthmore College, Swarthmore, Pennsylvania 19081
}

When Ss were simultaneously adapted to horizontal and to vertical target displacements of equal rate during head turning about a vertical axis, the adaptation effects measured by one-trial tests immediately after the adaptation period were about equal. But retests after a time lapse of 10 and $20 \mathrm{~min}$, during which $S$ sat immobile and with eyes closed, showed a greatly different rate of dissipation of the two adaptation effects. After a lapse of 20 min, the effect of adaptation to horizontal target displacements had been reduced to $37 \%$, whereas the effect of adaptation to vertical displacements at this final test still stood at $80 \%$ of the initial measurement. The decline over $20 \mathrm{~min}$ in the latter case was so smail that it could readily be ascribed to an effect of the two tests that preceded the final test. These two tests represented an effective exposure to natural viewing conditions and hence caused an unlearning of the adaptation, an effect whose existence we had demonstrated in previous work with the one-trial test.

One of the important issues in perceptual adaptation concerns the relationship between the learning process that causes the adaptation and the product of previous learning that governed S's perception prior to adaptation. The latter may be considered the result of an adaptation process also, namely, an adaptation to the natural conditions of viewing or listening, which is then temporarily superseded by experimental adaptation. In the end, after the postadaptation test has been administered, $S$, who is exposed again to the natural conditions, readapts to them, and his perceptual performance becomes normal again. There are, however, numerous instances of experimental adaptation where normalization of perception occurs without exposure to the natural conditions. A mere lapse of time during which $S$ remains passive and shut off from relevant stimulation suffices here to reestablish the normal perceptual function. It is obviously of great interest to know where such spontaneous dissipation of the effect of experimental adaptation occurs and in what kinds of adaptation it does not. It is, in fact, possible that all experimental adaptation dissipates spontaneously and that we deal here, rather, with differences in the rate at which perceptual functions return to normal. The experiment to be reported is part of a project where the rate of dissipation of a number of

*This work was supported by Grant 11089 from the National Institutes of Health to Swarthmore College, Hans Wallach, principal investigator. different kinds of adaptation effects is

The present experiment compares the rate of spontaneous dissipation of three kinds of adaptation to field displacements during head movements, field displacements in the direction of the head movements, either with or against it, or at right angles to the head movements. In previous work (Wallach, Frey \& Romney, 1969), we referred to the first two kinds of adaptation with the term "related to the constancy of visual direction," while we regarded the last one as unrelated to it. This distinction refers to the following considerations. With the visual field stationary, a head turning will cause a displacement of the field relative to the head. When an identical displacement is caused by moving the visual field, the displacement will be perceived as motion of the environment, but when it is caused by a head movement, the environment will appear stationary. This is due to a compensating process which we called constancy of visual direction, or CVD, which takes head movements into account in a most accurate manner where perceived motion or rest of the environment is concerned (Wallach \& Kravitz, 1968). The field displacements for which this constancy process compensates take place in the direction parallel to the plane in which the head turns, and that is the reason for calling adaptation to objective field displacement in this direction, i.e., to field displacements with or against the head movement, constancy related. When such objective field displacements are described as being studied. displacements relative to the turning head, they subtract themselves from or add themselves to the displacement that the objectively stationary environment undergoes relative to the turning head. It is the latter displacement that CVD causes to be perceived as environmental rest, whereas even a small increase or decrease in this displacement (caused by an objective field displacement against or with the head movement) is perceived as environmental motion. In other words, CVD singles out a particular rate of displacement parallel to the plane of the head rotation and makes it the zero point of the perceived environmental motions such that smaller rates of displacement produce perceived environmental motions in the direction with the head movement and larger rates cause perceived motions of the field against the head turning. The particular rate of displacement relative to the head that leads to perceived rest is the one produced by the stationary environment. Adaptation to natural viewing conditions, which causes the development of this way of perceiving motion and rest during $a$ head movement, is concerned with field displacements parallel to the plane of the head rotation, for that is the displacement of the stationary environment relative to the turning head. Experimental adaptation to objective field displacements parallel to the plane of the head rotation thus modifies the result of the adaptation to natural viewing conditions.

Experimental adaptation to field displacements at right angles to the plane of the head rotation is not so closely related to CVD. It amounts to a field displacement relative to the head that is oblique to the plane of the head rotation (namely, the resultant of the objective field displacement and the displacement of the stationary field relative to the turning head). Such an oblique displacement is not involved in the acquisition of CVD; it does not occur under natural conditions. Thus, experimental adaptation to field displacement parallel to the plane of the head rotation (Type A adaptation) modifies a product of a previously learned compensating process, while experimental adaptation at right angles to the plane of the head rotation (Type B adaptation) does not. When Type $B$ adaptation has developed, the adaptation to the natural viewing conditions that $S$ brings to the experiment still functions unaltered. For instance, partial adaptation to vertical field displacement during head turning in the horizontal plane causes that field displacement to be perceived at a smaller rate than the objective 
displacement would warrant, but the field motion that is seen is still in a vertical direction: CVD, which causes perceived field immobility as an alternative to perceived horizontal field motion, still functions fully. With the two types of adaptation thus differently related to the product of previous learning of CVD, it seemed possible that they differed with regard to spontaneous dissipation of the experimental adaptation. Wallach and Floor (1970) had obtained significant dissipation for Type A adaptation, which most likely was spontaneous, while the results for Type $B$ adaptation were not clear. ${ }^{1}$

\section{PROCEDURE}

The general plan of our experiment was to produce experimental adaptations to horizontal field displacement and to vertical field displacenent, both during head turning and to monitor their spontaneous dissipation. We measured adaptation effects three times, immediately after the adaptation period and 10 and $20 \mathrm{~min}$ later. Between tests, $S$ sat quietly, his eyes closed. For obvious reasons, we used the one-trial method introduced by Wallach and Frey (1969). This method measured the extent of the apparent motion which a stationary luminous spot in the dark underwent as an effect of adaptation when $S$ moved his head. This was done by combining the "subjective" motion caused by the adaptation effect with an objective motion of the target spot at right angles to the former. When the field displacement to which $S$ adapted was horizontal and, say, in the direction with the head movement, the apparent motion caused by the adaptation effect was against the head movement (to the left when the head turned right). When this apparent motion was combined with a vertical displacement of the target spot such that it moved objectively upward when the head turned right and down when the head turned left, the perceived target motion was oblique, slanting counterclockwise with respect to the vertical. It was this slant angle which $S$ reproduced in the test and which measured the adaptation effect. Apart from the ease with which Ss can make such slant estimates, the procedure has the following advantage: the extent of the apparent horizontal motion, which depends on the amount of adaptation achieved or, after partial dissipation, on the amount of residual adaptation effect, depends also on the extent of the head rotation. But since, because of the mechanism used to produce the objective vertical target displacement, the latter also varied with the extent of the head movement, the slant angle actually represented the ratio of the apparent horizontal target motion to the head movement. This measure therefore closely corresponded to the displacement ratio, that is, the ratio of the objective angular field displacement to the angle of the head rotation causing it, with which we had previously measured this kind of vertical displacements during head rotation in the horizontal plane (Type B adaptation), a stationary target would afterwards appear to move vertically, and this was combined with an objective horizontal target displacement. Here the estimated slant angle was measured as a deviation from the horizontal (Wallach, Frey, \& Romney, 1969).

It was important that the conditions for the two types of adaptation to be compared be identical. We achieved this by adapting simultaneously to horizontal and vertical field displacements of equal magnitude, a $40 \%$ displacement ratio. The apparatus described in detail by Wallach and Kravitz (1968), equipped with two mirrors to provide horizontal and vertical target displacements by Wallach and Frey (1969) and improved by Wallach and Floor (1970) was used. Simultaneous vertical and horizontal field displacements of equal displacement ratio (DR) produced an oblique target displacement of $45 \mathrm{deg}$ as resultant. There were two conditions of adaptation: the horizontal field displacement was either with or against the direction of the head rotation while there was only a single combination of head turning and vertical field displacement; when $S$ turned his head to the right, the target spot moved objectively upward, and when the head turned left the target moved down. Therefore, the resultant target motion was between upper right and lower left in the case of the horizontal displacement with the head the horizontal displacement was against the rotation of the head. During the adaptation period, S remained attached to the test apparatus which was set to produce the simultaneous vertical and horizontal target displacements just described when he turned his head adaptation. 2 When $S$ adapted to movement and of opposite slant when

back and forth. The adaptation training consisted of 200 such cycles of head rotation.

Adaptation to the horizontal displacement component was tested by presenting $S$ with an objective vertical target displacement of $40 \%$ DR, and a horizontal target displacement of $40 \%$ DR was used to test for Type B adaptation. Slant settings were made in the manner described by Wallach and Frey (1969). As in our previous work, tests were administered before and after the adaptation period. At the outset of the experiment, Ss were given practice with slant estimation by following the procedure described by Wallach and Frey (1969) under "Preliminary Experiments (2)"; Ss who performed poorly in this kind of test were eliminated at this point. ${ }^{3}$

Twenty Ss completed the experiment. For half of them, the test for adaptation to the horizontal displacement component always preceded the test for the Type B adaptation in the pre- and postadaptation tests as well as in the tests after the two dissipation periods. For the other half, the order of the two tests was always reversed. Half of the 10 Ss that made up each group adapted to horizontal target displacement in the direction with the head movement and the other half against it.

\section{RESULTS}

The results are given in Table 1 , where the listed mean slant settings are computed from the differences between the respective post- and preadaptation scores. The effects measured immediately after the adaptation period were approximately the same for the two types of adaptation. 4 The loss in adaptation effect after two 10 -min periods during which $\mathbf{S}$ sat with eyes closed was very different for the two types of adaptation. In the case of the adaptation to horizontal target displacement, there was a consistent decline in the measured effect. Nineteen out of 20 Ss gave a smaller slant estimate after the first $10-\mathrm{min}$ time lapse than immediately after the adaptation period, and the individual adaptation effects remaining after the

Table 1

Mean Effects of Adaptation to Oblique Target Displacement After 200 Head Movements and After Two Periods of Dissipation

\begin{tabular}{lccccc}
\hline & \multicolumn{2}{c}{ Type A Adaptation } & & \multicolumn{2}{c}{ Type B Adaptation } \\
\cline { 2 - 3 } & $\begin{array}{c}\text { In Deg } \\
\text { of Slant }\end{array}$ & $\begin{array}{c}\text { Percent of } \\
\text { Full Effect }\end{array}$ & & $\begin{array}{c}\text { In Deg } \\
\text { of Slant }\end{array}$ & $\begin{array}{r}\text { Percent of } \\
\text { Full Effect }\end{array}$ \\
\hline After 200 HM & 13.60 & 100 & 14.88 & 100 \\
10 Min Later & 8.50 & 63 & 14.15 & 95 \\
20 Min Later & 5.05 & 37 & 11.85 & 80 \\
\hline
\end{tabular}


second 10-min time lapse were all smaller than those measured after the first 10-min period of dissipation. The effect of adaptation to vertical displacement during head turning declined much more slowly. The first 10-min lapse caused only a $5 \%$ mean decrease in the effect, which was not significant $(p>.1)$. The decline of $20 \%$ for the total dissipation period of $20 \mathrm{~min}$ was significant $(\mathrm{p}<.001)$. The differences in the residual effects for Type $A$ and Type $B$ adaptation after time lapse were highly significant. There was only one $S$ who failed to give a larger slant estimate in the test for adaptation to vertical displacement than in the other test after the $10-\mathrm{min}$ dissipation period, and, after the 20-min time lapse, measured residual Type $B$ adaptation was larger for every one of the $20 \mathrm{Ss}$ than the effect remaining from adaptation to horizontal target displacement.

That the $20 \%$ loss in Type B adaptation effect after the 20-min time lapse represents spontaneous dissipation seems questionable. Under very similar conditions, Wallach, Frey, and Romney (1969) found an almost identical loss upon immediate retesting. In their first experiment demonstrating adaptation to vertical target displacement during head turning, a test immediately following an adaptation period consisting of 100 head movements yielded an effect on slant estimation of $6.04 \mathrm{deg}$. A second test, which differed from the first only in the sense of the objective target displacement relative to the head turning, measured an effect of $4.70 \mathrm{deg}, 78 \%$ of the effect yielded by the first test. Since the second test followed the first test immediately, this loss represents the effect of the first test. It must be ascribed to the nature of the one-trial test employed, which measured an apparent vertical displacement of a target that was objectively stationary insofar as the vertical dimension was concerned. (The objective horizontal displacement of the test target, which served to measure the extent of the apparent vertical displacement as a slant, was here irrelevant.) Observing a stationary target during a head movement, however, amounted to an exposure to natural viewing conditions and should cause partial undoing of the effect of experimental adaptation.

One might find difficulty in interpreting the loss in the effect of adaptation to vertical target displacement, which we found to amount to only $5 \% 10 \mathrm{~min}$ after the first test and to $20 \% 10 \mathrm{~min}$ after the second test in terms of the result of Wallach, Frey, and Romney just reported, on the ground that the latter obtained a loss of $22 \%$ without any time delay and after only one test. Two points should be considered here. The previous authors employed experimental adaptation amounting to 100 head movements, whereas we used 200 ; hence, the same test, consisting in an exposure to what amounts to an unlearning condition, could be expected to have a smaller effect in the latter case. And then there is the possibility that an analogue to reminiscence ${ }^{5}$ for the effect of experimental adaptation operated to augment this effect when the test was delayed and thus counteracted the loss due to the unlearning effect of the preceding test.

At any rate, Wallach, Frey, and Romney unquestionably obtained from a one-trial test an unlearning effect that exceeded in magnitude the decrease in the adaptation effect with the time lapse we obtained for Type B adaptation, and that makes it likely that this decrease was due to the intervening test or tests (two tests preceded the last one) and not to the time lapse as such.

\section{DISCUSSION}

Our experiment had two results: Type A adaptation in CVD dissipated spontaneously, and Type $B$ adaptation probably did not, and if it did, it did so much more slowly than Type A adaptation. For the spontaneous dissipation of Type A adaptation we offer the following explanation: We make the plausible assumption that CVD, the way of perceiving that $S$ brings to our adaptation experiment, is also the product of an adaptation process, an adaptation to the natural viewing conditions, and that, as a learning product, it survives in the nervous system the experimentally produced adaptation while the latter dominates S's perception. As during the dissipation period, the encounters with the conditions that produced experimental adaptation gradually recede into the past, CVD, the product of adaptation to the natural viewing conditions, becomes more and more manifest in S's perception, which thus reverts to CVD: experimental adaptation gradually dissipates. This interpretation of spontaneous dissipation of Type $A$ adaptation is supported by results obtained by Wallach and Floor (1970). They found that if CVD, that is, adaptation to natural viewing conditions, was strengthened just before experimental adaptation began, by exposing $S$ for 200 head movements to a stationary target, experimental adaptation of Type A was retarded. This showed that CVD is, indeed, the product of an adaptation process and that this process interacts with experimental adaptation. Spontaneous dissipation is simply another result of such interaction.

It would be pleasant if it were possible to ascribe the failure of Type $B$ adaptation to dissipate to the fact that, prior to experimental adaptation, no learning product existed of such a nature that it would be replaced by Type $B$ adaptation. Such an assumption is, in fact, quite plausible. It seems, indeed, that there should be no learning connected with the fact that under natural viewing conditions the visual field does not move upward when the head turns right and down when it turns left. But this explanation is refuted by another result obtained by Wallach and Floor: Type $B$ adaptation, too, is retarded when $S$ is exposed for 200 head movements to a stationary target prior to experimental adaptation. Here, too, exposure to the natural viewing conditions just before experimental adaptation interfered with that adaptation process. Thus, it cannot be argued that exposure to natural viewing conditions leaves no learning effect that Type B adaptation would have to supersede. Instead, an explanation for the failure of Type $B$ adaptation to dissipate spontaneously is needed that is compatible with the fact that practice with the natural viewing conditions interferes with Type B adaptation. What is here practiced under natural viewing conditions, must be different in nature from the learning process responsible for CVD, because the spontaneous dissipation found in the case of Type $A$ adaptation does not occur in Type $B$ adaptation. And that practice must be of such a nature that it can interfere with the process of vertical target displacement, because that practice subsequently retards such adaptation.

There seems to be only one kind of learning that fits these criteria, and that is connected with the eye movements that occur when one turns his head and looks at a point in the stationary visual field. Such eye movements are normally horizontal, but when, during Type $B$ adaptation, the target is vertically displaced during head turning these eye movements become slanted. 6 In the experiment by Wallach and Floor, having $S$ look at a stationary target during head turning just before adaptation to vertical target displacement amounted to practicing horizontal eye movements during head turning, and this, in fact, retarded adaptation to vertical target displacement, which involves slanted eye movements. It thus seems that learning to make oblique eye movements during head turning instead of horizontal ones is part of Type $B$ adaptation. 
Making horizontal eye movements during head turning is practiced throughout life. So is CVD, the adaptation to the natural conditions of having the visual field displaced relative to the head during head turning at the normal rate. In fact, the individual occasions for both practices are the same; each turning of the head provides one. Yet, the interaction between the two practices and the effects of experimental adaptation differs. Type A adaptation dissipates rapidly and S's perception reverts to CVD without practice. Experimental adaptation to vertical target displacement does not dissipate spontaneously or, if it does, reverts to normal much more slowly.

\section{REFERENCES}

WALLACH, H., \& FLOOR, L. On the relation of adaptation to field displacement during head movements to the constancy of visual direction. Perception \& Psychophysics, 1970, 8,
95-98.

WALLACH, H., \& FREY, K. J. Adaptation in the constancy of visual direction measured by a one-trial method Perception \& Psychophysics, 1969,5, 249-252.

WALLACH, H.. FREY, K. J., \& ROMNEY, G. Adaptation to field displacement during head movement unrelated to the constancy of visual direction. Perception \&s Psychophysics, 1969. 5. 253-256.

WALLACH, H.. \& KRAVITZ, J. $H$. Adaptation in the constancy of visual direction tested by measuring the constancy of auditory direction. Perception \& Psychophysics, 1968. 4, 299-303.

\section{NOTES}

1. The dissipation measured for Type $B$ adaptation was actually smaller than that obtained for Type $A$ adaptation, but the difference failed to be significant.

2. For a detailed explication of the displacement ratio measure, see Wallach and Kravitz (1968).

3. Ss who prior to adaptation gave slant-estimation scores with more than three reversals were eliminated.

4. Two directions of target displacement were used in the case of Type $A$ adaptation. Ten of our $S s$ adapted to horizontal target displacements $w$ ith the head movernents and 10 in the direction against them. No significant differences in adaptation or dissipation were obtained for these conditions. Previously, Wallach and Frey (1969) and allach, Frey, and (1969) and again Wallach, Frey, and of adaptation when the field displacernents were in the direction with the head movement. Then, the same $S$ s were, at different times, employed in the two conditions. In our experiment, with conditions. In our experiment, with
different $S$ s used in the two conditions and with only 10 Ss in each group. the difference failed to become manifest.

5. A discussion of reminiscence may be found in C. E. Osgood, Methods and Theory in Experimental Psychology. New York: Oxford University Press, 1953, pp. 509-513

6. This is so because an objectively vertical displacement, when vectorially added to the horizontal displacement relative to the turning head of an objectively relative to the turning head of an objectively
stationary point, amounts to a displacement relative to the head that is oblique to the plane of the head rotation.

(Accepted for publication March 28, 1971.) 